Pierre-Yves TESTENOIRE

Université Paris 3 / HTL

\title{
Ce que les théories du discours doivent à Saussure
}

\section{Introduction}

Le présent article se propose d'interroger l'usage qui est fait des textes de Ferdinand de Saussure dans les théories contemporaines du discours. La notion de discours en linguistique étant, on le sait, plurivoque ${ }^{1}$, nous entendrons ici par «théorie du discours » les approches qui s'intéressent à la dimension transphrastique des énoncés linguistiques et aux conditions socio-historiques de leurs productions. Cette acception large des «théories du discours » couvre un champ évidemment immense traversé par des orientations théoriques diverses, des querelles terminologiques et conceptuelles, qu'il est impossible d'embrasser. Ces approches, dont l'historiographie actuelle situe l'émergence entre les années 30 et les années 50, se sont développées depuis en se réclamant de théoriciens divers selon la perspective envisagée-Voloshinov, Bakhtine, Harris, Halliday, Benveniste, Austin, Althusser, Foucault, ...--, mais rarement de Saussure.

Pour exposer l'interrogation qui est à l'origine de cet article, confrontons deux séries de jugements portés sur le rôle de la pensée saussurienne dans la formation du concept moderne de discours. Les trois premiers datent du milieu des années 70 et sont empruntés à trois ouvrages d'initiation aux théories linguistiques :

a) Toute l'élaboration théorique de Saussure repose sur sa définition de l'objet langue dont l'étude suppose un double rejet: celui de l'histoire et celui de la réalité objective (entre autre sociale). [...] L'extension, peut-être illégitime, de la démarche saussurienne a fait l'objet, par ailleurs, d'une contestation théorique, qui remet précisément en cause le double rejet que nous mentionnons plus haut et vise à substituer aux dualités saussuriennes dont il dérive, une problématique théorique, articulant le champ de la linguistique à des domaines comme l'histoire, la pratique sociale, l'idéologique (voir Haroche, Henry, Pêcheux 1971). Cette nouvelle problématique se caractérise par le rejet de l'idée d'un code neutre

\footnotetext{
${ }^{1}$ Voir par exemple les différentes acceptions de discours en linguistique distinguées par Maingueneau (1976 : 11-12) et reprises dans Charaudeau \& Maingueneau (2002 : 185-187).
} 
(la langue) opposé à la liberté individuelle du locuteur (la parole) (Fuchs \& Le Goffic 1975 : 16).

b) Dans cette perspective [celle de Saussure], il n'y a pas de place pour le « discours », concept qui vise à déposséder le sujet parlant de son rôle central pour l'intégrer au fonctionnement d'énoncés, de textes dont les conditions de possibilités sont systématiquement articulées sur des formations idéologiques (Maingueneau $1976: 6$ ).

c) Par rapport à l'œuvre de Saussure et des structuralistes dans leur ensemble, les travaux de Benveniste témoignent d'une indéniable originalité. Ils ont en effet mis en évidence l'existence d'un « autre niveau de l'analyse linguistique », celui du fonctionnement discursif du sujet, et démontré que ce fonctionnement ne peut être décrit dans les termes classiques de l'analyse sémiologique (Bronckart 1977 : 306).

Ces trois passages ont en commun de présenter le concept de discours en linguistique comme une nouveauté par rapport à la linguistique de Saussure, que cette nouveauté provienne de la sémantique discursive de Pêcheux (texte a), de l'analyse du discours à la Foucault (texte b) ou de la théorie de l'énonciation de Benveniste (texte c). Ces présentations dans des manuels avalisent les débats qui président au développement des théories du discours en France dans la décennie qui précède. Elles ne rendent cependant pas compte à quel point le concept de discours se forge chez Benveniste, Pêcheux (1990 [1971]) ou Slakta (1971) par une discussion serrée des concepts saussuriens, et spécifiquement de la distinction langue-parole (Voir Maldidier 1993, Puech 2005, Angermuller 2013). Le projet interdisciplinaire d'analyse du discours se conçoit alors comme une modalité d'appréhension des extérieurs de la langue face à la clôture du système attribuée aux linguistiques d'inspiration saussurienne. Le discours apparaît donc dans ces présentations des années 75-77 comme un concept nouveau en linguistique, acquis par rapport à la pensée saussurienne selon des modalités qui vont du simple dépassement (texte c), à la contestation (texte a), ou à la tabula rasa (texte b).

Sur cette même problématique, voici maintenant, trois autres jugements, formulés une trentaine d'années plus tard :

d) Les Écrits de linguistique générale récemment découverts permettent de lire un autre Saussure que celui du Cours de Bally \& Sechehaye (1916), un autre que celui des Sources de Godel (1957), et que celui de l'édition critique d'Engler (1967-1974). C'est un penseur du primat du discours qu'on découvre, avant Benveniste et autrement (Meschonnic $2005: 10$ ). 
e) La linguistique saussurienne reste révolutionnaire parce que c'est une linguistique du texte et non une linguistique du signe (Rastier $2010: 328$ ).

f) Comme en attestent de multiples passages de son œuvre, Saussure a soutenu clairement que la dimension de l'activité de discours était première eu égard à la langue (Bota \& Bronckart 2010 : 341).

Ces trois citations, comme les précédentes, sont délibérément empruntées à des orientations de recherche diverses. Elles convergent cependant pour faire du discours, non plus un au-delà de la linguistique saussurienne, mais bien un élément de sa pensée théorique. Ces trois jugements témoignent d'un déplacement dans l'horizon de rétrospection de certains théoriciens de la discursivité ou de la textualité. Les citations mises en regard dessinent donc sur une période d'une trentaine d'années, une évolution sensible, voire un renversement, dans l'appréciation de ce que le concept de discours doit à Saussure. Ce sont les modalités de cette évolution qui sont l'objet du présent article. Comment est-on passé, et selon quelles voies, en l'espace de trente ans, d'un Saussure ignorant la catégorie du discours ou même entravant sa conceptualisation, à un Saussure penseur de la discursivité, voire de son primat?

\section{2. Évolutions et seuils de rupture rétrospectifs}

L'un des facteurs de cette évolution semble être ce qu'on a pu appeler la progressive "grammaticalisation » des théories du discours opérée dans les années 80 (Courtine 1991), grammaticalisation qui s'est couplée avec une désidéologisation. Saussure et son concept de langue, perçu comme a-social et a-historique, perdent alors un peu leur statut d'épouvantail réactionnaire coupable de faire obstacle à l'avènement d'une linguistique matérialiste $^{2}$. Un autre facteur - en tout cas l'un des plus explicitement exprimés - est l'élargissement, à la fin du $\mathrm{XX}^{\mathrm{e}}$ siècle, du corpus des textes autographes de Saussure disponibles. C'est ce que semble indiquer Henri Meschonnic (texte d), mais d'une façon nettement paradoxale, puisqu'il appuie sa thèse d'un primat du discours chez Saussure dans la suite de son article non pas sur les nouveaux documents publiés dans les Écrits de linguistique générale (désormais $E L G$ ) mais sur les textes saussuriens que Meschonnic cite et connaît depuis les années 70.

\footnotetext{
${ }^{2}$ Sur l'idéologie réactionnaire attribuée à la pensée saussurienne dans la décennie 70 , on se reportera par exemple à Guespin (1971) ou à Calvet (1975) qui se réclame de la critique marxiste de Saussure développée par Voloshinov.
} 
L'évolution de certaines bibliographies sur la période considérée permet à la fois de confirmer et de nuancer cette hypothèse. En observant, par exemple, les différentes moutures des livres de Jean-Michel Adam sur la linguistique textuelle, il ressort que le chapitre introductif des Éléments de linguistique textuelle de 1990 ne mentionne pas Saussure parmi les théoriciens fondateurs d'une approche linguistique du texte. La référence à Saussure fait son apparition dans la version de 1999, Linguistique textuelle : des genres de discours aux textes, et gagne ensuite en importance, puisque l'ouvrage de 2005, La linguistique textuelle, s'ouvre sur cette référence avec un chapitre intitulé : "Introduction à l'analyse textuelle des discours : (Re)partir de Saussure, de Benveniste et de Bakhtine ». Les travaux de François Rastier illustrent sur la même période une évolution similaire : Saussure est absent de la bibliographie de Sémantique Interprétative (1987) et de Sens et textualité (1989), avant de devenir, une quinzaine d'années plus tard, une des références majeures de ses travaux.

C'est donc, semble-t-il, dans la dernière décennie du siècle que l'œuvre de Saussure a progressivement été considérée comme contenant une pensée productive du discours. Dans ce processus, les travaux de Simon Bouquet et Johannes Fehr du milieu des années 90 sur les écrits autographes du linguiste et les articles de Béatrice Turpin (1995 et 1996) sur les occurrences de «discours» chez Saussure, semblent avoir joué un rôle déterminant. La publication des $E L G$ quelques années plus tard, qui réunit des manuscrits édités dans les années 50 et 60 par Robert Godel et Rudolf Engler et d'autres découverts en 1996, n'agit dès lors pas comme une révélation, contrairement à ce qu'écrit Meschonnic, mais comme un manifeste brandi à titre rétrospectif pour transformer en rupture dans la réception de Saussure ce qui relève d'une évolution antérieure. Car ce ne sont pas les nouveaux manuscrits publiés dans les ELG-ceux de L'Essence double - qui alimentent la réévaluation de la dimension discursive de la pensée saussurienne. Elle est d'abord portée par une relecture des anciens documents - cahiers d'étudiants, notes de cours, notes Item-, rendue attentive, non pas tant à la notion de discours, mais à l'adjectif discursif qui donne lieu chez Saussure à des emplois substantivés («le discursif» (Saussure 2002: 95, 105, 117, 118; 1968/1974: 2061) ${ }^{3}$ ) ou à des syntagmes dont on redécouvre l'intérêt : «langue discursive » (Saussure 2002: 117), «langage discursif » (Ibid.: 95), « unités discursives » (Saussure 1968/1974: 1998), «ordre discursif» (Ibid. : 1985, 2186)... Un texte surtout va fédérer cette relecture du discursif chez Saussure : ce texte, on a pris l'habitude de l'appeler la «note sur le discours ». Le titre est

\footnotetext{
${ }^{3}$ Les renvois aux cahiers d'étudiants des Cours de linguistique générale se font selon la numérotation de l'édition d'Engler.
} 
étranger à Saussure ; il a été choisi par les éditeurs des $E L G$ et a joué un rôle déterminant pour la réception de ce texte ${ }^{4}$. Il est remarquable que les différentes perspectives que nous avons mentionnées - linguistique textuelle d'Adam, poétique de Meschonnic et de ses disciples, sémantique des textes de Rastier, ou interactionnisme socio-discursif de Bronckart - se réclament toutes à cette même période d'une pensée saussurienne du discours via cette note. Si cette communion est remarquable, le texte présente des caractéristiques propices à un tel succès.

Le texte en question fait partie de l'ensemble des cahiers d'anagrammes, considéré dans son extension maximale. À ce titre, il a été publié la première fois, non dans les Sources manuscrites de Robert Godel, mais dans Les Mots sous les mots de Jean Starobinski (1971: 14). Il a été rééditée en 1989 par René Amacker parce que «le lecteur intéressé par la théorie linguistique de Saussure ne songe peut-être pas à [l']aller chercher dans un livre sur les anagrammes » (1989: 93). Il a ensuite été intégré aux $E L G$, où il est classé par erreur parmi les «nouveaux documents (fonds BPU 1996) » (Saussure 2002 : 275), alors qu'il fait partie de l'ancien fonds des manuscrits. Ce texte est passé inaperçu jusqu'au milieu des années 90 , où il a fait l'objet d'une réévaluation intensive (Kim 1993: 67, Fehr 1995, Dessons $1995: 211-213$, Adam $1999: 23-29$ et 2001, Rastier $2003: 46-47$, Pétroff $2004: 141$, Suenaga $2005: 214-218, \ldots$ ), un numéro de la revue Langages (Chiss \& Dessons 2005) allant jusqu'à lui être presque entièrement consacré.

La note dite sur le discours constitue, avec le texte sur la consécutivité et «sur la moyenne des impressions acoustiques hors du temps» (Starobinski 1971 : 46-47), le second texte des Mots sous les mots dont la portée a été reçue comme touchant directement la théorie linguistique de Saussure. Le succès de ces deux textes, encore que différent ${ }^{5}$, tient d'une

\footnotetext{
${ }^{4}$ Les étapes du figement de la locution «note sur le discours » peuvent être reconstituées : Starobinski parle d' « un texte isolé, [qui] pose le problème [du discours] » (1971); Amacker (1989) et Fehr (1995) parlent d'une «note de F. de Saussure concernant le "discours" », Adam (2001 : 202) de «la note dite "sur le discours" »; Engler et Bouquet, enfin, dans les ELG choisissent le titre « Note sur le discours », sans signaler par des crochets, comme ils le font dans le reste de l'édition, que ce titre leur est dû. Ce titre, s'il est commode, introduit une indication de genre (Note sur...) et, par l'emploi systématique du déterminant défini (il y a pourtant d'autres notes sur le discours chez Saussure), confère au texte une exceptionnalité et une clôture artificielles.

${ }^{5}$ Alors que l'intérêt pour la note dite sur le discours a été différé aux années 90, l'engouement pour le texte sur la consécutivité a été immédiat. Dès la parution des premiers textes relatifs aux anagrammes, Jakobson, Lacan, Starobinski ou Wunderli soulignent l'importance de ce texte qu'ils opposent au principe de linéarité exposé dans le CLG. En 1970, Thomas Aron note déjà : « "Hors de l'ordre dans le temps qu'ont les éléments" est sans doute la phrase des cahiers
} 
part, à leur dimension spéculative, marquée formellement, dans les deux cas - et ce n'est pas un hasard - par des phrases interrogatives ; il tient, d'autre part, à leur isolement. Un texte d'une page, «décotextualisé », se prête en effet plus aisément à des raccourcis et à des reprises sous forme de formules que 50 pages des Notes Item ou 300 pages d'Essence double. Or, nous avons montré ailleurs comment la prise en compte de l'ensemble du cahier dans lequel le texte sur la consécutivité s'inscrit permettait de construire une autre interprétation que celle communément admise (Testenoire 2012). C'est cette même démarche que nous proposons d'appliquer pour la note dite sur le discours.

Cette note, par l'importance qu'elle a prise dans l'exégèse saussurienne ces dernières années, mérite qu'on s'y arrête d'un point de vue philologique. Pour l'arracher à son isolement, nous entendons considérer cet écrit comme relevant précisément d'un acte de discours qui s'inscrit dans une situation d'énonciation analysable d'après ses marques formelles et d'après ses éléments co-textuels. Il s'agit, en somme, de lire la note dite sur le discours dans sa discursivité. Pour cela, une description informée du manuscrit saussurien conservé à la Bibliothèque de Genève s'avère indispensable.

\section{Que faire de la note dite sur le discours?}

La note est écrite sur la dernière page d'un cahier de 20 pages sans titre classé, dans les fonds de la Bibliothèque de Genève, sous une cote intitulée «Métrique Védique» (BGE, Ms. fr. 3961/10). Sous cette appellation, Robert Godel, qui a effectué le premier classement des manuscrits saussuriens, a réuni 26 cahiers et une liasse de feuillets. Cet ensemble, si l'on ajoute trois autres cahiers réunis sous d'autres cotes, constitue l'intégralité des manuscrits connus relatifs aux recherches métricophoniques sur le Véda, que l'on rattache communément à la préoccupation anagrammatique. Ces manuscrits sont majoritairement dévolus à l'hypothèse de l'homophonie, hypothèse selon laquelle «tout se répond d'une manière ou d'une autre dans les vers» (Saussure 2013: 118). Consacrées à l'inventaire des phonèmes dans chaque strophe, les analyses de ces cahiers se présentent principalement sous forme de tableaux. La note dite sur le discours est écrite sur la dernière page d'un de ces cahiers consacrés à la parité phonique dans le Véda. Dans cet ensemble, la page sur le discours reste toutefois isolée d'un point de vue conceptuel, puisque les concepts qu'interroge la note dite sur le discours (langue, discours,

saussuriens la plus souvent citée. Ce faux alexandrin mallarméen recèle un singulier pouvoir » (Aron $1970: 57$ ). 
signification...) ne sont pas mobilisés en tant que tels dans les cahiers, mais aussi d'un point de vue formel, puisque la discursivité propre à la note fait figure d'exception. Dans les pages du cahier qui précèdent la note, le syntagmatique se réduit à peu de chose. Ainsi l'inscription dans ces cahiers ne permet pas d'inférer une relation entre la réflexion sur le discours et la comptabilisation phonique du Véda. En revanche, elle aide à préciser sa datation, dont les commentateurs se contentent souvent de dire qu'elle est incertaine. La relation du cahier avec l'hypothèse de l'homophonie permet de situer un terminus post quem assuré : celui de 1906. Un passage d'une lettre de Saussure à Meillet (Benveniste 1964 : 113) confirme qu'une partie de ces cahiers a été rédigée cette année-là. D'autres documents récemment découverts (voir Testenoire 2013 : 108) prouvent qu'une autre partie de ces cahiers date du second semestre de 1909. L'écriture de la page sur le discours peut donc être située dans un empan temporel qui va de 1906 à 1909, peut-être début 1910, qui correspond à la période où Saussure dispense ses deux premiers cours de linguistique générale.

Venons-en maintenant au texte de la note :

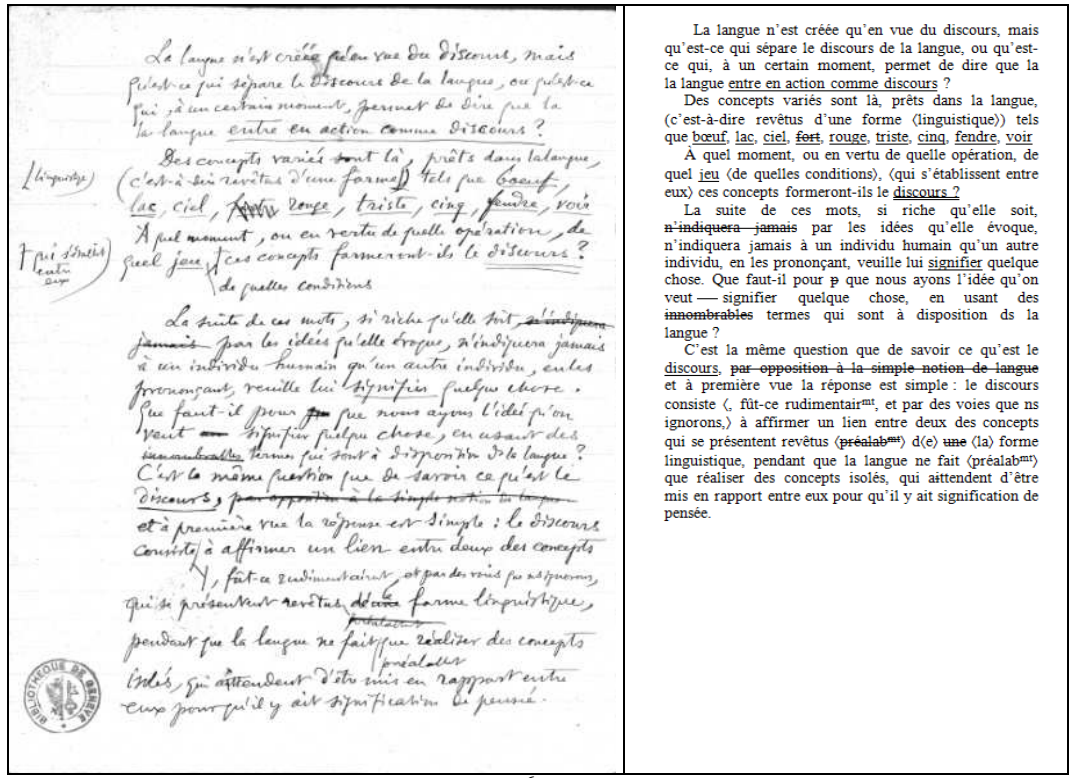

Figure 1 : BGE, Ms. fr. 3961/10, f $20 \mathrm{v}^{\circ 6}$ ${ }^{6}$ On remarquera que notre transcription se démarque de celle d'Amacker (1989) qui rend par
des majuscules le double soulignement de «discours ». Or Saussure utilise de façon 
Le questionnement qui se fait jour dans les premiers paragraphes, qui ont focalisé l'attention des critiques, est celui de l'articulation entre langue et parole développée dans les cours de linguistique générale, articulation située à l'endroit de flottement qu'est, dans la théorie saussurienne, la syntaxe. La difficulté est qu'il n'est à aucun moment question de parole, mais bien de discours. Or, comme l'ont remarqué plusieurs commentateurs (par exemple Godel (1957: 259) ou Turpin (1996: 264)), discours et discursif sont fréquemment employés dans les deux premiers cours, mais sont absents du troisième. Dans les deux premiers cours, discours a deux emplois : soit apposé à parole, de manière synonymique ${ }^{7}$, soit employé concurremment à elle lorsqu'il est question de chaîne, ou d'agencement syntagmatique $^{8}$. Ainsi, l'adjectif discursif opposé à intuitif est proposé, dans le deuxième cours, comme variante pour qualifier les rapports syntagmatiques opposés aux associatifs (Saussure 1968-1974 : 2061). Il y a donc bien un flottement terminologique, qui paraît stabilisé dans le troisième cours où discours n'est pas évité par hasard et où parole prend alors les deux dimensions qu'on lui connaît: phonation et exécution individuelle (Bouquet 1997 : 338-seq.). Ce qui frappe, dans ce cadre, est la similitude entre cette note et un passage, bien connu par ailleurs, du deuxième cours. Voici ce qu'on trouve dans les cahiers d'Albert Riedlinger à une leçon de janvier 1909 :

D'une part, il existe le trésor intérieur qui équivaut aux casiers de la mémoire : c'est là ce qu'on peut appeler le magasin ; c'est un des 2 lieux, une des deux sphères. C'est dans ce trésor qu'est rangé tout ce qui peut entrer en activité dans le second lieu. Et le second lieu, c'est le discours, c'est la chaîne de la parole. Suivant qu'on se place dans l'un ou l'autre 〈lieu d'existence des mots〉 nous aurons affaire à des groupes, mais à des groupes de nature $\langle$ tout à fait $\rangle$ différente :

\begin{tabular}{c|l} 
Trésor (magasin) & Discours Chaîne \\
\hline Unités d'association & $\begin{array}{l}\text { Unités discursives } \\
\text { (c'est-à-dire qui se produisent dans le } \\
\text { discours) }\end{array}$ \\
Groupes au sens de familles & Groupes au sens de syntagmes
\end{tabular}
(Saussure 1968-1974 : II R 89 [1998])

différenciée la fonction d'emphase des majuscules et du soulignement. La transcription des $E L G$ est, quant à elle, fautive puisqu'elle transcrit sans rature l'adjectif «fort » qui est incontestablement barré.

${ }^{7}$ Voir Saussure (1968-1974 : 2561) : «Pour que cette forme pénètre dans la langue, il faut que $1^{\mathrm{e}}$ quelqu'un l'ait improvisée, et $2^{\mathrm{e}}$ improvisée à l'occasion de la parole, du discours. » Voir aussi Saussure (Ibid. : 2560).

${ }^{8}$ Cet emploi se rencontre surtout dans le deuxième cours : voir Saussure (1968-1974 : 1731, 1998, 2020, 2039, 2061, 2087). 
Les deux textes articulent les concepts de langue et de discours avec des similitudes évidentes : la métaphore du $\operatorname{stock}^{9}$ et la formule «entre en action», d'un côté, «entre en activité » de l'autre. La différence entre «activité » et «action»a son importance: Wunderli (1988) a montré qu'action, chez Saussure, peut être tenu pour un hyperonyme d'acte et d'activité mais désigne aussi le produit de cette activité. La « langue entrant en action comme discours» devient à la fois ergon et energeia ${ }^{10}$. La seconde distinction majeure entre ces textes réside dans les prépositions « dans » et « comme », l'une créant de la contigüité quand l'autre crée de la disjonction.

Il est chronologiquement possible qu'un de ces deux textes soit la source de l'autre. Ce qu'il importe surtout de noter c'est que les efforts de certains critiques, pas toujours philologiquement bien informés, pour révéler, à partir de la note dite sur le discours, un concept saussurien de discours totalement disjoint de celui de parole repose avant tout sur une approche immanente des textes. La notion de discours présente chez Saussure ne recouvre certes pas celle de parole retenue dans le $C L G$ (voir Parret 1971). Cependant opposer cette notion au «couple langue parole hérité de la réception de Saussure par la linguistique structurale » (Dessons 2006 : 207) ne résiste pas à l'analyse du corpus saussurien pris dans sa globalité. C'est méconnaître à la fois la temporalité de la pensée saussurienne - son energeia précisément -, et le statut spécifique des textes qui la consignent.

Ce qui, en définitive, fait l'intérêt de cette note, certainement contemporaine du deuxième cours, et qui explique son succès, ce n'est pas une conceptualisation du discours, qui reste à l'état d'interrogation, mais la formulation problématisée et dynamique de l'articulation entre langue et parole. Ce dynamisme passe à la fois par la formule «la langue entre en action comme discours » et par le statut manuscrit du texte, dynamisme que perd l'exposé didactique du cours nécessairement plus figé, avec sa présentation tabulaire et son image des sphères. Cependant l'interpénétration des deux sphères, que l'on redécouvre à l'occasion de la réévaluation de cette note, est attestée dans bien d'autres passages des cours (Saussure 1968-1974 : 341-342, 344, 1640-1641).

\footnotetext{
${ }^{9}$ On trouve dans d'autres manuscrits, les notes dites Item, la même articulation entre le « trésor mental » et le «discours » au moyen du concept de «langue discursive » (voir Saussure $2002: 95$ et 117).

${ }^{10}$ Dans son enquête terminologique, Wunderli (1988) n'examine toutefois pas ces deux passages.
} 


\section{Le réinvestissement discursif des légendes et des anagrammes}

Un autre élément, révélateur de l'évolution du rôle de la discursivité dans la réception saussurienne, est le changement dans le traitement des recherches dites parfois périphériques aux cours de linguistique générale : les légendes et les anagrammes. L'évolution accompagne celle observée préalablement : le discours s'impose comme catégorie pour penser l'un ou l'autre de ces travaux, parfois les deux, à partir du milieu des années 90. Ainsi Gérard Dessons considère que « la théorie des anagrammes constitue en fait une approche de la signification dans la parole, c'est-à-dire de la notion de discours » (1995: 211). Cette interprétation est partagée par Béatrice Turpin, pour qui il s'agit d' " un discours sur le discours qui vient compléter la bipartition langue/parole » (1995: 306), ou par Jean-Michel Adam qui estime qu'avec les anagrammes « on passe de la logique du signe à l'ordre du discours » (2001: 215). Le travail sur les légendes est pour Cristian Bota et Jean-Paul Bronckart «une longue démarche relevant indiscutablement de l'analyse de discours » (2010 : 197). François Rastier, enfin, écrit que ces deux recherches « auront fait quitter la problématique du signe pour celle du texte, tant dans son expression - les anagrammes - que dans son contenu - les motifs et symboles » (2003: 47). Or, ce prisme est absent de la première réception de ces travaux, c'est-à-dire des interprétations de Starobinski, de Wunderli, de Jakobson ou même de Meschonnic et d'Adam sur les anagrammes, d'Avalle, d'Engler et de Prosdocimi sur les légendes, pour ne citer que les plus consistantes. Toutes évaluent le déplacement sémiotique opéré par ces travaux par rapport aux conceptions du signe héritées du $C L G$; aucune n'en fait alors un espace de réflexion sur la discursivité et la textualité. Mais là encore, le processus n'est pas directement corrélé à l'élargissement du champ des textes saussuriens. L'essentiel des manuscrits sur les légendes est rendu public entre le milieu des années 70 et des années 80 (Avalle 1973, Saussure 1986). Quant au corpus des anagrammes édité, il est stabilisé en 1971 avec les Mots sous les mots et n'évolue que de façon marginale ensuite (voir Saussure 2013 : 15-seq.). Comme pour les écrits de linguistique générale, l'insistance sur la problématique de la discursivité dans les légendes et les anagrammes résulte moins de la découverte de nouveaux textes que d'un changement de point de vue.

La catégorie du discours qui émerge au milieu des années 90 dans la réception critique de ces textes permet de les réinvestir dans une problématique linguistique. De ce point de vue, le concept de discours contribue au passage d'une lecture oppositive des différentes recherches de Saussure à une lecture intégrative. La réception immédiate des cahiers d'anagrammes s'est principalement effectuée, on le sait, dans les marges de 
la linguistique - chez les littéraires, les poéticiens, les psychanalystes -, suivant en cela le geste inaugural de Robert Godel déléguant l'analyse des manuscrits d'anagrammes à son collègue de littérature française de l'université de Genève, Jean Starobinski. Quant aux lectures linguistiques, elles ont fait de ces travaux une contestation du Saussure structuraliste attribué au $C L G$. Avec le motif, bien connu, des «deux Saussure » qui a cours jusqu'au début des années 80 , prévaut la thèse selon laquelle les anagrammes contrediraient la théorie saussurienne du signe et ses principes fondamentaux : subversion du signifié par le signifiant, remise en cause de l'arbitraire, de la linéarité... En corrélant les légendes et les anagrammes à une pensée du discours restituée via la note dite sur le discours, ces travaux sont interprétés comme porteurs non plus de la négation du signe, mais de son dépassement. Quel que soit le nom que l'on donne à cet au-delà du signe - signifiance si l'on se réclame de Benveniste, texte si l'on se réclame de Coseriu - l'avenir des cours de linguistique générale serait ainsi inscrit en gésine dans les manuscrits. Cette approche holiste du travail saussurien ne constitue pas à proprement parler une révolution ; il consiste à penser, non plus sur le mode du conflit mais sur celui du changement d'échelle détermination du global sur le local chez Rastier, passage du phrastique au transphrastique chez Adam-, l'hiatus maintenu entre, d'une part, l'enseignement de linguistique générale et, d'autre part, le travail sur les anagrammes et sur les légendes.

\section{Conclusion}

Les réévaluations de la discursivité ou de la textualité chez Saussure apparues depuis le milieu des années 90 prouvent que l'héritage saussurien continue de jouer un rôle de conscience disciplinaire, tout du moins pour la linguistique de langue française. Dans la formation d'une «matrice disciplinaire qui peut être réinvestie, transformée, étendue et contestée » (Puech 2013 [en ligne]), toutefois, le corpus des manuscrits saussuriens en partie disponible tend à remplacer progressivement le $C L G$. Le déplacement des textes de référence se traduit dans le mode de lecture qui en est fait. La réception s'opère dès lors sur les béances du corpus saussurien, prenant appui sur les textes questionnants ou inachevés (la note dite sur le discours) ou invoquant les recherches encore largement inédites (les anagrammes). Elle se caractérise aussi par le téléologisme adopté et, à des degrés divers, revendiqué : Saussure permettrait une pensée du discours dans la mesure où on saurait la faire fructifier. La pluralité des approches contemporaines de la discursivité permet alors de reconstruire des filiations diverses qui 
convergent, par des chemins distincts, au maître genevois : passant tantôt via Propp et l'analyse structurale des récits ${ }^{11}$, tantôt via Benveniste $^{12}$, tantôt via Hjelmslev et Coseriu ${ }^{13}$... Pour reprendre le cheminement présenté en introduction, il semble que l'on soit passé en l'espace d'une trentaine d'années d'un rapport dialectique à Saussure - le Pour et Contre Saussure de Louis-Jean Calvet - à ce qu'il faut bien appeler une forme d'œcuménisme. Que le discours soit le lieu et l'instrument de cette communion en dit peut-être plus long sur la linguistique contemporaine que sur Saussure.

\section{Références bibliographiques}

ADAM, J.-M., (1990), Éléments de linguistique textuelle. Théorie et pratique de l'analyse textuelle. Bruxelles : Mardaga.

- (1999), Linguistique textuelle : des genres de discours aux textes. Paris : Nathan.

- (2001), «Discours et interdisciplinarité : Benveniste lecteur de Saussure », Cahiers Ferdinand de Saussure, 54, 201-218.

- (2005), La linguistique textuelle. Paris : Armand Colin.

AMACKER, R., (1989), « Note de Ferdinand de Saussure concernant le discours », Cahiers Ferdinand de Saussure, 43, 93-94.

ANGERMÜLLER, J., (2013), Analyse du discours poststructuraliste. Limoges : Lambert-Lucas.

ARON, Th., (1970), «Une seconde révolution saussurienne ? », Langue française, 7, 56-62.

BENVENISTE, E., (1964), «Lettres de Ferdinand de Saussure à Antoine Meillet », Cahiers Ferdinand de Saussure, 21, 93-130.

BOUQUET, S., (1997), Introduction à la lecture de Saussure. Paris : Payot.

BRONCKART, J.-P., (1977), Théories du langage. Bruxelles : Mardaga.

BRONCKART J.-P., \& BOTA, C., (2010), «Dynamique et socialité des faits langagiers ». In BRONCKART, J-P., BULEA, E., \& BOTA, C., (éds.), Le projet de Ferdinand de Saussure. Genève : Droz, 193-213.

CALVET, L.-J., (1975), Pour et contre Saussure. Paris : Payot.

CHARAudeau, P., \& MAINGUENEAU, D., (éds.) (2002), Dictionnaire d'analyse du discours. Paris : Seuil.

CHAROLLES, M., \& COMBETTES, B., (1999), «Contribution pour une histoire récente de l'analyse du discours », Langue française, 121, 76-115.

\footnotetext{
${ }^{11}$ Voir Avalle (1973) et Bronckart (2010).

${ }^{12}$ Voir Adam (2001), Meschonnic (2005) et Dessons (2005). On connaît le programme développé dans Critique du rythme : Saussure, par son concept de valeur, rendrait possible une pensée du discours que Benveniste a développée, lui-même, par son concept de discours, rendant possible une pensée du rythme que Meschonnic se charge de développer (1982: 29seq.). L'intérêt de Meschonnic et de ses élèves pour la note dite sur le discours à partir des années 90 s'explique dans ce dispositif : elle vient corroborer a posteriori ce téléologisme autocentré.

${ }^{13}$ Rastier (2003, 2010)
} 
CHISS, J.-L., \& DESSONS, G., (éds.) (2005), Linguistique et poétique du discours à partir de Saussure, Langages, 159.

COURTINE, J.-J., (1991), «Le discours introuvable: marxisme et linguistique (1965-1985) », HEL, 13(2), 153-171.

DESSONS, G., (1995), Introduction à la poétique. Paris : Dunod.

- (2006), Émile Benveniste, l'invention du discours. Paris : In Press.

FEHR, J., (1995), « Boeuf, lac, ciel — concierge, chemise, lit », Linx [En ligne], 7. Consulté le 10 juillet 2013 : < http://linx.revues.org/1236>.

FUCHS, C., \& LE GOFFIC, P., (1975), Initiation aux problèmes des linguistiques contemporaines. Paris : Hachette.

GODEL, R., (1957), Les Sources manuscrites du cours de linguistique générale. Genève : Droz.

GUESPIN, L., (1971), «Problématique des travaux sur le discours politique », Langages, 23, 3-24.

HAROCHE, C., HENRY, P., PÊCHEUX, M., (1971) «La sémantique et la coupure saussurienne : langue, langage, discours », Langages, 24, 93-106.

KIM, S.-D., (1993), «La mythologie saussurienne: une nouvelle vision sémiologique? (À propos de la continuité de la pensée saussurienne) », Semiotica, 97, 5-78.

MAINGUENAU, D., (1976), Initiation aux méthodes de l'analyse du discours. Paris : Hachette.

MALDIDIER, D., (1993), «L'inquiétude du discours. Un trajet dans l'histoire de l'analyse du discours: le travail de Michel Pêcheux », Semen [En ligne], 8. Consulté le 11 juillet 2013 : < http://semen.revues.org/4351 >.

MESCHONNIC, H., (1982), Critique du rythme. Paris : Verdier.

- (2005), «Saussure ou la poétique interrompue », Langages, 159, 10-18.

PARRET, H., (1971), Language and Discourse. The Hague : Mouton \& co.

PÊCHEUX, M., (1990) [1971], L'inquiétude du discours, édition MALDIDIER, D. Paris : Édition des Cendres.

PÉTROFF, A.-J., (2004), Saussure: la langue, l'ordre et le désordre. Paris : L'Harmattan.

PUECH, C., (2005), « L'émergence de la notion de discours en France et les destins du saussurisme », Langages, 159, 93-110.

- (2013), «L'esprit de Saussure : réception et héritage (l'héritage linguistique saussurien: Paris contre Genève) », Les dossiers de HEL, 3 [supplément électronique à la revue Histoire Épistémologie Langage]. Consulté le 3 février 2015 : < http://htl.linguist.univ-paris-diderot.fr/num3/puech.pdf >

RASTIER, F., (2003), «Le silence de Saussure ou l'ontologie refusée », Cahier de l'Herne, 76, 23-51.

- (2010), «Saussure et la science des textes ». In BRONCKART, J.-P., BULEA, E., \& BOTA, C., (éds.), Le projet de Ferdinand de Saussure. Genève : Droz, 315-335.

SAUSSURE, F., de, (1968-1974), Cours de linguistique générale, édition critique ENGLER, R. Wiesbaden : Otto Harrassowitz.

- (1986), Le Leggende Germaniche, édition MARINETTI, A. \& MELI, M. Este : Zielo. 
— (2002), Écrits de linguistique générale, édition ENGLER, R. \& BOUQUET, S. Paris : Gallimard.

- (2013), Anagrammes homériques, édition TESTENOIRE, P.-Y. Limoges: Lambert-Lucas.

SLATKA, D., (1971), «Esquisse d'une théorie lexico-sémantique : pour une analyse d'un texte politique (cahiers de doléances) », Langages, 23, 87-134.

SUENAGA, A., (2005), Saussure, un système de paradoxes : langue, parole, arbitraire et inconscient. Limoges : Lambert-Lucas.

TESTENOIRE, P.-Y., (2012), «La linéarité saussurienne en rétrospection», Beiträge zur Geschichte der Sprachwissenschaft, 22, 149-170 repris dans Texto! Textes \& Cultures [en ligne], 19(2). Consulté le 3 février 2015: $<$ http://www.revue-texto.net/index.php?id=3503 >.

- (2013), Ferdinand de Saussure à la recherche des anagrammes. Limoges: Lambert-Lucas.

TURPIN, B., (1995), «Discours, langue et parole. Une comparaison entre la réflexion sur les anagrammes et les études sur les légendes », Linx [En ligne], 7. Consulté le 12 juillet 2013 : < http://linx.revues.org/1173>.

- (1996), «Discours, langue et parole dans les cours et les notes de linguistique générale de F. de Saussure », Cahiers Ferdinand de Saussure, 49, 251-266.

WUNDERLI, P., (1988), «"Acte", "Activité" und "action" bei Saussure », Cahiers Ferdinand de Saussure, 42, 175-201.

Résumé : Cet article porte sur l'usage qui est fait des textes de Ferdinand de Saussure dans les théories contemporaines du discours. On étudie l'évolution de ces quarante dernières années dans la réception de l'œuvre saussurienne selon le prisme de la discursivité et de la textualité. On interroge les modalités de lecture des textes du corpus saussurien - note dite "sur le discours », anagrammes, légendes - qui fondent la reconnaissance, historiquement récente, d'une pensée du discours chez Saussure.

Mots-clés : réception ; historiographie ; discursivité ; textualité ; manuscrits

Abstract : This article focuses on the use made of Ferdinand de Saussure's texts in contemporary theories of discourse. It examines the evolution of the last forty years in the reception of the Saussurian work through the prism of discursivity and textuality. It questions the readings of the texts of the Saussurian corpus - note called "on discourse", anagrams, legends - that are at the basis of the historically recent recognition of a thought on discourse by Saussure.

Keywords : reception ; historiography ; discursivity ; textuality ; manuscripts 